\title{
Neurologisch wachsam bleiben
}

\section{In MMW 21-22/2015 ging es ab S. 57 um neurologische Schlüsselsymp- tome. Ein Leser beschreibt, wie schnell man diese übersehen kann:}

_ Am ersten Arbeitstag nach meinem Urlaub kam ein langjährig bekannter, 59-jähriger Patient zu mir und berichtete, vor drei Wochen zu Hause über einen Wäschekorb gestürzt zu sein. Erstdiagnose: V. a. Commotio cerebri. Seitdem Leistungsminderung, jetzt geringes Schlurfen, diskrete Koordinationsstörungen, subjektiv Zittrigkeit. Meine Urlaubsvertretung wertete dies bei blandem Verlauf als Commotio-Folgen.

Ich fand den Sturz zu Hause ungewöhnlich. Angesichts des schlurfenden Gangs und des Tremors veranlasste ich ein Schädel-MRT am Folgetag. Der Ra- diologe rief mich mit dem Befund eines $22-24 \mathrm{~mm}$ großen peritentoriellen Meningeoms und beginnenden Hirndruckzeichen an. Einen Tag später wird der Patient zur OP stationär aufgenommen.

M. E. ist den Kollegen in der Vertretung kein Fehler vorzuwerfen. Sie kannten den Patienten nicht lange genug und stellten die naheliegendste Verdachtsdiagnose.

- Dr. med. Thomas G. Schätzler, Facharzt für Allgemeinmedizin, Dortmund (über springermedizin.de)

\section{KORREKTUREN}

In MMW 18/2015 ging es auf S. 14 um das richtige Anlegen einer Thoraxdrainage. Dabei ist uns ein kleiner Fehler unterlaufen. Nach unserer Schilderung sollte man sich bei der Inzision am Oberrand des Intercostalraums vorarbeiten, weil sich dort wenige Gefäße und Nerven befinden. Gemeint war der Oberrand der Rippe.

In MMW 18/2015 ging es ab S. 56 um die intravenöse Behandlung der Eisenmangelanämie. Auch vor dem Hintergrund der aktuellen Fachinformationen verändern sich die Angaben zur Dosierung von Eisenisomaltosid (vgl. Tab. 3). Die korrekte Bezeichnung ist jetzt Eisenderisomaltose. Dieses Präparat ist Dextran-basiert, die minimale Infusionsdauer der maximalen Einzeldosis ist aktuell 15-30 min, die minimale Injektionsdauer der maximalen Einzeldosis ist aktuell $2 \mathrm{~min}$ (oder $250 \mathrm{mg} / \mathrm{min}$ ).

\section{Hier steht eine Anzeige.}

